Revue québécoise de psychologie

$W_{\text {québécolise }}^{\text {Revue }}$

\title{
LE SOUTIEN SOCIAL, LA DÉTRESSE PSYCHOLOGIQUE, LE BIEN-ÊTRE ET LE FONCTIONNEMENT SCOLAIRE DE JEUNES LESBIENNES, GAIS ET BISEXUELS (LGB) QUÉBÉCOIS SOCIAL SUPPORT, PSYCHOLOGICAL DISTRESS, WELL-BEING AND SCHOOL FUNCTIONING IN YOUNG LESBIANS, GAYS, AND BISEXUALS (LGB)
}

\author{
Marie-Hélène Létourneau-Picard, François Sallafranque St-Louis et Isabelle \\ Green-Demers
}

Volume 37, numéro 1, 2016

URI : https://id.erudit.org/iderudit/1040110ar

DOI : https://doi.org/10.7202/1040110ar

Aller au sommaire du numéro

Éditeur(s)

Revue québécoise de psychologie

ISSN

2560-6530 (numérique)

Découvrir la revue

Citer cet article

Létourneau-Picard, M.-H., Sallafranque St-Louis, F. \& Green-Demers, I. (2016). LE SOUTIEN SOCIAL, LA DÉTRESSE PSYCHOLOGIQUE, LE BIEN-ÊTRE ET LE FONCTIONNEMENT SCOLAIRE DE JEUNES LESBIENNES, GAIS ET BISEXUELS (LGB) QUÉBÉCOIS. Revue québécoise de psychologie, 37(1), 203-224. https://doi.org/10.7202/1040110ar
Résumé de l'article

L'objectif du présent projet était d'examiner les associations entre le soutien de la famille et des amis, le rapport à l'orientation sexuelle, la détresse, le bien-être et le fonctionnement scolaire de jeunes lesbiennes, gais et bisexuel(le)s québécois $(n=29)$. Les résultats ont révélé que le soutien de la famille était négativement associé au rapport conflictuel à l'orientation sexuelle ainsi qu'à la détresse et positivement associé au bien-être. Le soutien des amis était associé négativement à la peur de la violence homophobe et positivement au bien-être. Le rapport conflictuel à l'orientation sexuelle était associé positivement à la détresse et négativement au bien-être. Le soutien des enseignants était positivement associé à la motivation scolaire et négativement à la violence homophobe, cette dernière étant négativement associée à la motivation. 


\title{
LE SOUTIEN SOCIAL, LA DÉTRESSE PSYCHOLOGIQUE, LE BIEN- ÊTRE ET LE FONCTIONNEMENT SCOLAIRE DE JEUNES LESBIENNES, GAIS ET BISEXUELS (LGB) QUÉBÉCOIS
}

SOCIAL SUPPORT, PSYCHOLOGICAL DISTRESS, WELL-BEING AND SCHOOL FUNCTIONING IN YOUNG LESBIANS, GAYS, AND BISEXUALS (LGB)

\author{
Marie-Hélène Létourneau-Picard ${ }^{1}$ \\ Université du Québec en Outaouais \\ François Sallafranque St-Louis \\ Université du Québec en Outaouais \\ Isabelle Green-Demers \\ Université du Québec en Outaouais
}

La recherche de l'identité, à l'adolescence, implique de nombreux questionnements. L'un de ceux-ci, à savoir vers quel genre l'attirance physique est la plus importante, touche beaucoup de jeunes. Alors que la majorité de la population sait qu'elle est hétérosexuelle, d'autres se définissent comme étant homosexuels ou bisexuels, tandis que d'autres sont en questionnement concernant leur orientation sexuelle. Selon Birkett, Espelage et Kœnig (2009), 10,5\% des 7376 étudiants de $7^{\mathrm{e}}$ et $8^{\mathrm{e}}$ année de leur échantillon seraient des jeunes lesbiennes, gais ou bisexuels (LGB) alors que 4,6\% seraient incertains de leur orientation sexuelle. King et ses collègues (2008) ont observé que $5,6 \%$ des 226315 étudiants de leur échantillon s'identifiaient comme étant LGB. La prévalence varie dans une certaine mesure d'une étude à l'autre, car plusieurs jeunes LGB sont incertains ou ont peur de se révéler. Une étude effectuée auprès d'adolescents québécois rapporte que $3 \%$ des jeunes de l'échantillon s'identifient comme LGB, $3 \%$ sont en questionnement et $6 \%$ se considèrent hétérosexuels, tout en ayant une attirance, des fantasmes ou des comportements sexuels avec des personnes du même genre (Zhao, Montoro, Igartua, \& Thombs, 2010). Par ailleurs, Savin-Williams (1995) remarque qu'il y aurait plus de personnes ayant des comportements sexuels avec d'autres du même genre que les statistiques l'indiquent, puisque seuls 27 à $30 \%$ des participants qui rapportaient régulièrement des désirs ou des conduites homosexuelles s'identifiaient comme LGB. Prise dans son ensemble, l'information qui précède nous apprend qu'un nombre non négligeable de jeunes du Québec ou d'ailleurs sont LGB ou s'interrogent sur leur orientation sexuelle.

La société nord-américaine en général et la société québécoise en particulier ont beaucoup évolué au fil des dernières décennies et les attitudes envers la diversité sexuelle sont devenues plus libérales. Or,

1. Adresse de correspondance: Marie-Hélène Létourneau-Picard, a/s Isabelle GreenDemers, Département de psychoéducation et de psychologie, Université du Québec en Outaouais, C.P. 1250, succ. Hull, Gatineau (QC), J8X 3X7. Courriel : mh_letourneau@hotmail.com 
malgré ce changement, les LGB font malheureusement encore face à des préjugés et à des comportements hostiles qui peuvent aller jusqu'à la violence homophobe. Les jeunes LGB constituent ainsi une population qui fait face à des défis importants qui diffèrent de ceux de leurs pairs hétérosexuels et il appert pertinent de s'intéresser à divers éléments clés de leur vécu. Le présent projet a pour objectif d'examiner le soutien social de la famille et des amis, le rapport à l'orientation sexuelle, la détresse psychologique, le bien-être et le fonctionnement scolaire de jeunes LGB québécois. Les sections qui suivent recensent la documentation à ces divers sujets. Les objectifs spécifiques et les hypothèses de ce projet sont détaillés par la suite.

\section{SOUTIEN SOCIAL}

L'influence bénéfique du soutien social a été abondamment documentée (pour des recensions, veuillez consulter Tang, Pu, \& Yao, 2008 ou Roehrle \& Strouse, 2008). Le soutien des personnes significatives de l'environnement agit à titre de facteur de protection contre la détresse psychologique et favorise le développement et le maintien du bien-être (Lazarus, 1990; Lazarus \& Folkman, 1984). Ceci est aussi vrai chez les jeunes LGB, où les figures clés de l'environnement social en général sont les gens de la famille et les amis.

Lorsque le système familial est constructif et fonctionnel, il s'agit d'une source de soutien importante pour les adolescents. Même si lors de cette phase du développement les amis sont appelés à jouer un rôle de plus en plus important, la famille demeure l'unité sociale d'origine de l'adolescent. Les interactions positives avec les membres de la famille contribuent au développement de l'identité et de l'estime personnelle (Anderson, 1998; Bregman, Malik, Page, Makynen, \& Lindahl, 2013). Quand le milieu familial remplit son rôle naturel de soutien, il est également source de sécurité et de réconfort en cas de détresse (Ainsworth, Blehar, Waters, \& Wall, 1978; Bowlby, 1969; 1973; Mikulincer \& Shaver, 2007). Ces fonctions développementales de la famille sont susceptibles d'être particulièrement nécessaires pour les jeunes LGB qui font face au défi personnel de transiger avec une orientation sexuelle qui s'écarte de la norme ainsi qu'aux défis sociaux que la diversité sexuelle peut poser. En effet, la documentation révèle que le soutien des parents a un impact bénéfique sur le processus identitaire des jeunes LGB (Bregman et al., 2013; Floyd, Stein, Harter, Allison, \& Nye, 1999) et sur leur santé mentale (Hershberger \& D'Augelli, 1995). À l'inverse, l'absence de soutien ou le rejet des parents a des conséquences négatives sur l'acceptation de l'orientation sexuelle et sur la santé mentale qui peuvent aller jusqu'au développement de troubles dépressifs et au suicide (Rothman, Sullivan, Keyes, \& Boehmer, 2012; Ryan, Huebner, Diaz, \& Sanchez, 2009). 
Les amis sont également une source de soutien potentielle importante chez les jeunes LGB. Le soutien et l'acceptation des pairs ont été associés positivement au degré de confort avec l'orientation sexuelle (Mustanski, Newcomb, \& Garofalo, 2011; Sheets \& Mohr, 2009) et au bien-être psychologique (Berghe, Dewaele, Cox, \& Vincke, 2010; Bos, Sandfort, Bruyn, \& Hakvoort, 2008). Au contraire, l'absence de soutien et le rejet ont été associés à la psychopathologie et au risque de suicide (Hatzenbuehler, 2011; Sheets \& Mohr, 2009).

\section{RAPPORT À L'ORIENTATION SEXUELLE}

L'orientation homosexuelle est définie comme une identification à « une prépondérance de sentiments sexuels ou érotiques, de pensées, de fantasmes et de comportements désirés avec des membres du même sexe » (Savin-Williams, 1990). Cette prépondérance se retrouve chez les bisexuels face aux membres des deux sexes (Anhalt \& Morris, 1998). Les jeunes LGB font face à plusieurs difficultés lors du processus d'intégration de leur orientation sexuelle. Dans le cadre de ce projet, une attention particulière a été accordée à trois composantes psychologiques qui témoignent d'un rapport conflictuel ou tourmenté à l'orientation sexuelle : la confusion, le besoin d'acceptation et l'homophobie internalisée.

La confusion réfère à l'incertitude qu'une personne a envers sa propre orientation sexuelle. Particulièrement au début de l'adolescence, un grand nombre de jeunes sont incertains de leur orientation sexuelle (Balsam \& Mohr, 2007; Birkett et al., 2009), ce qui pourrait être expliqué par la pression à adopter une identité sexuelle plus conforme aux normes sociales (Eliason, 1996). Les personnes qui sont indécises par rapport à leur orientation sexuelle et les personnes bisexuelles présentent plus de symptômes psychologiques négatifs (Birkett et al., 2009; Russel, Seif, \& Truong, 2001; Saewyc, Homma, Skay, Bearinger, Resnick,\& Reis, 2009). En contraste, la clarté de l'identité est liée à un meilleur bien-être psychologique (King \& Smith, 2004; Rosario, Schrimshaw, \& Hunter, 2011).

Le besoin d'acceptation est un facteur de vulnérabilité de l'identité LGB proposé par Mohr et Fassinger (2000). Ce besoin signifie que l'acceptation de l'orientation sexuelle est conditionnelle à celle d'autrui. II reflète une dépendance à l'opinion des gens et exprime la peur du jugement social. II s'agit d'un déficit d'autonomie dans l'acceptation personnelle qui est alimenté par la prévalence des normes hétérosexuelles et des préjugés sociaux à l'égard des LGB. Le besoin d'acceptation au sujet de l'orientation sexuelle a été associé négativement à l'estime de soi (Mohr \& Fassinger, 2000). 
L'homophobie internalisée est une vision négative de soi, accompagnée d'émotions néfastes dirigées vers soi ( $p$. ex., honte, dégoût), suscitées par l'orientation sexuelle (Benibgui, 2011). En raison des attentes hétérosexuelles de la société et des expériences fréquentes de discrimination, l'homophobie internalisée toucherait une grande partie des personnes LGB (Balsam \& Mohr, 2007; Weibley, 2010). L'homophobie internalisée a été associée négativement au bien-être psychologique et positivement aux troubles dépressifs et anxieux ainsi qu'aux idéations suicidaires (Bricker, 2009; Feinstein, Wadsworth, Davila, \& Goldfried, 2014; Igartua, Gill, \& Montoro, 2003; Newcomb \& Mustanski, 2010; OrtizHernández, 2005).

\section{DIFFICULTÉS PSYCHOLOGIQUES ET BIEN-ÊTRE CHEZ LES LGB}

Au cours de la période de l'adolescence et du début de l'âge adulte, un consensus dans la documentation exprime que les jeunes faisant partie de minorités sexuelles souffrent plus fréquemment de problèmes psychologiques que les adolescents hétérosexuels (Birkett et al., 2009; Bos et al., 2008; King et al., 2008; Hospers \& Jansen, 2005). Il appert donc important de s'attarder à cet aspect de leur vécu. Dans le cadre de ce projet, trois éléments de détresse psychologique ont été examinés: l'anxiété, la dépression et les inquiétudes reliées à la violence homophobe. Afin d'être en mesure de dresser un portrait équilibré qui comprend également des composantes positives, quatre aspects complémentaires du bien-être psychologique ont aussi été considérés, soit l'expérience d'émotions positives, la satisfaction envers la vie, la perception (locus) de contrôle et l'estime de soi.

\section{Détresse psychologique}

\section{Anxiété}

Certaines études suggèrent que le pourcentage de jeunes LGB qui présentent des symptômes et des troubles anxieux est plus élevé que le pourcentage de jeunes hétérosexuels (Bostwick, Boyd, Hughes, \& McCabe, 2010; D'Augelli \& Hershberger, 1993; Spencer \& Patrick, 2009). Des taux de prévalence accrus chez les personnes LGB ont été identifiés, entre autres, pour le trouble d'anxiété généralisé (Fergusson, Horwood \& Beautrais, 1999) et l'anxiété sociale (Potoczniak, Aldea, \& DeBlaere, 2007).

\section{Dépression}

Les jeunes LGB sont également plus susceptibles de vivre des troubles dépressifs, d'entretenir des idéations suicidaires et d'effectuer des tentatives de suicide que les adolescents hétérosexuels (Goodenow, Szalacha, \& Westheimer, 2006; Liu \& Mustanski, 2012; Warner et al., 
2004). Par exemple, une méta-analyse révèle que le pourcentage moyen de comportements suicidaires est de $28 \%$ chez les jeunes LGB et de $12 \%$ chez les jeunes hétérosexuels (Marshal et al., 2011). À la suite d'une tentative de suicide, les LGB continuent de surcroît à vivre un haut niveau de détresse longtemps après avoir posé leur geste (Rosario, Schrimshaw, \& Hunter, 2005).

\section{Inquiétudes liées à la violence homophobe}

L'intimidation, le caïdage et la violence verbale ou physique sont des réalités pour bien des jeunes. De plus, les adolescents LGB font quotidiennement face au stress accru de faire partie d'une minorité sexuelle souvent stigmatisée. Ce contexte social difficile peut être source d'inquiétudes d'être victime de discrimination, de menaces, de vandalisme et de violence verbale ou physique (D'Augelli et al., 2005; D'Augelli, Pilkington \& Hershberger, 2002).

\section{Bien-être psychologique \\ Émotions positives et satisfaction envers la vie}

Si plusieurs problèmes psychologiques ont été identifiés chez les LGB, certaines études ont rapporté que les participants LGB vivent plus d'émotions positives que d'émotions négatives (Knight \& Hope, 2012; Lee, Dean, \& Jung, 2008) et dénotent un niveau modérément élevé de satisfaction envers la vie (Lee et al., 2008).

\section{Locus de contrôle}

Le locus de contrôle est défini par le degré auquel la personne croit que les conséquences de ses comportements sont déterminées par des facteurs internes ou externes (Rotter, 1990). II représente ainsi la perception de la capacité à agir sur l'environnement. Lorsque le locus de contrôle est interne, la perception du pouvoir d'action est élevée. Le locus de contrôle est un indicateur révélateur du bien-être psychologique (p. ex., Infurna, Gerstorf, Ram, Schupp, \& Wagner, 2011). Une étude réalisée auprès de LGB a révélé que l'internalité du locus de contrôle est négativement associée à la détresse causée par la discrimination (Carter, Mollen, \& Smith, 2014).

\section{Estime de soi}

L'estime de soi correspond au sentiment de valeur personnelle et c'est un élément central classique du bien-être psychologique (Rosenberg, 1965). L'estime de soi est considérée comme une composante importante de la santé mentale (Hershberger \& D'Augelli, 1995). II serait particulièrement utile de s'attarder à cette composante du bien-être psychologique chez les jeunes LGB, car certaines études ont révélé un 
niveau plus faible d'estime de soi chez ces derniers (Spencer \& Patrick, 2009; Bos et al., 2008).

\section{FONCTIONNEMENT SCOLAIRE}

Parce que l'école est un milieu important où évoluent les jeunes LGB, trois variables scolaires ont été examinées à titre complémentaire dans le cadre de ce projet. II s'agit du soutien des enseignants, de la motivation scolaire et de la violence homophobe vécue à l'école.

Les enseignants sont les principales figures d'autorité et les principaux agents de socialisation avec lesquels les élèves interagissent à l'école (Green-Demers, Mageau, \& Pelletier, 2013). Ils ont donc beaucoup d'impact sur la qualité de leur expérience dans ce milieu. Le soutien des enseignants a été associé à la motivation scolaire des élèves. Cet effet a été abondamment documenté (voir Guay, Ratelle, \& Chanal, 2008, ou Reeve \& Jang, 2006, pour des recensions).

De son côté, la motivation scolaire est un des meilleurs indicateurs d'un fonctionnement harmonieux à l'école. La motivation est un antécédent proximal direct de l'effort et de l'attention investis en classe (Reeve, 2002; Schunk \& Zimmerman, 2008), elle prédit la réussite et la persévérance scolaires (Green-Demers et al., 2013; Ratelle, Guay, Vallerand, Larose, \& Sénécal, 2007) et c'est un facteur de protection contre le décrochage (Alivernini \& Lucindi, 2011). II y a peu d'information sur la motivation scolaire des jeunes LGB, quoique les résultats d'une étude québécoise suggèrent que l'expérience de l'homophobie à l'école favorise une diminution de la motivation et une augmentation de l'absentéisme (Martin \& Beaulieu, 2002).

Le soutien des enseignants est également négativement associé à la violence homophobe vécue à l'école (Goodenow et al., 2006; Harris, 1997). En effet, malheureusement, l'école est un milieu qui peut être propice à la discrimination (D'Augelli et al., 2005), à l'intimidation (Birkett et al., 2009) et à la violence homophobe verbale et physique (Harris, 1997; Teasdale \& Bradley-Engen, 2010; Warner et al., 2014). Récemment, en 2012 , le Québec s'est doté du projet de loi $n^{\circ} 56$, c'est-à-dire la Loi visant à prévenir et à combattre l'intimidation et la violence à l'école qui est en vigueur dans chaque établissement scolaire (Assemblée nationale Québec, 2012). Elle s'inscrit dans la Stratégie gouvernementale de mobilisation de la société québécoise afin de lutter contre l'intimidation et la violence à l'école qui doit notamment inclure des mesures de prévention pour contrer toute forme d'intimidation chez les personnes des minorités sexuelles. II est donc possible que cette loi ait amené une diminution des taux de violence homophobe rapportés par les jeunes LGB québécois. 


\section{LA RECHERCHE SUR LES ADOLESCENTS LGB AU QUÉBEC}

Parce que les attitudes relatives à l'orientation sexuelle peuvent beaucoup changer d'un milieu à l'autre, il est important de situer le contexte socioculturel dans lequel prennent place les études à ce sujet. Le Québec se distingue de la majorité des autres provinces du Canada et des états américains par ses attitudes plus libérales sur le plan de la sexualité et de l'orientation sexuelle (Langis, Germain, Dallaire, Normandeau, \& Ross, 2015). La recherche sur les minorités sexuelles est également particulièrement florissante au Québec (p. ex., Igartua et al., 2003; Julien \& Chartrand, 2005; Lavoie \& Côté, 2014; Richard, 2015).

Par le passé, peu d'attention a cependant été accordée aux adolescents LGB québécois. Un certain nombre d'études portant sur cette population ont toutefois été réalisées très récemment. Des exemples de thèmes examinés par cette documentation en émergence incluent l'orientation sexuelle des jeunes québécois (Igartua, Thombs, Burgos, \& Montoro, 2009; Zhao et al., 2010), les antécédents relationnels et les conséquences psychologiques de la révélation de l'orientation sexuelle des adolescents LGB à leurs parents (D'Amico, Julien, Tremblay \& Chartrand, 2012), la détresse psychologique chez les jeunes LGB (D'Amico et al., 2012; Zhao et al., 2010) ainsi que l'homophobie, la violence homophobe verbale et physique et l'inclusion de la diversité sexuelle à l'école (Chamberland, Émond, Julien, Otis, \& Ryan, 2010; Peter, Taylor, \& Chamberland, 2015; Petit, Chamberland, Richard, \& Chevrier, 2011). Le présent projet s'inscrit dans la foulée de ces travaux novateurs.

\section{OBJECTIF ET HYPOTHĖSES}

Ce projet vise à examiner les interrelations entre le soutien des parents et des amis, le rapport à l'orientation sexuelle, la détresse psychologique, le bien-être et le fonctionnement scolaire d'adolescents LGB québécois. Les hypothèses avancées sont les suivantes: (1) le soutien des parents et des amis est négativement associé à un rapport tourmenté à l'orientation sexuelle (confusion, besoin d'acceptation, homophobie internalisée); (2) le soutien des parents et des amis est négativement associé à la détresse psychologique (symptômes anxieux et dépressifs, inquiétudes liées à la violence homophobe) et positivement associé au bien-être (émotions positives, satisfaction envers la vie, locus de contrôle et estime de soi); (3) le rapport conflictuel à l'orientation sexuelle est positivement associé à la détresse psychologique et négativement associé au bien-être; (4) le soutien des enseignants est positivement associé à la motivation scolaire et négativement associé à la violence homophobe vécue à l'école; (5) la violence homophobe vécue à l'école est négativement associée à la motivation scolaire. 


\section{MÉTHODOLOGIE}

\section{Participants et procédure}

L'échantillon comprend 29 participants LGB francophones et inclut 12 garçons, 15 filles, un transgenre et un participant qui n'a pas dévoilé son sexe biologique. Sur le plan de l'orientation sexuelle, il y avait 8 personnes lesbiennes, 11 gais, 8 bisexuel(le)s (1 garçon et 7 filles) et 2 données manquantes. Chez les garçons, l'identité sexuelle rapportée était masculine $(n=6)$ ou androgyne $(n=6)$. Aucun d'entre eux n'a rapporté d'identité sexuelle féminine. Chez les filles, l'identité sexuelle était féminine $(n=7)$, androgyne $(n=6)$ ou masculine $(n=1)$. L'identité sexuelle comportait également trois données manquantes. Les participants étaient âgés de 14 à 21 ans $(M=18,69, E . T .=2,04)$. Dix-huit d'entre eux étudiaient à temps plein, 5 à temps partiel et les autres n'étaient pas aux études. Cinq participants travaillaient à temps plein, 12 à temps partiel et les autres ne détenaient pas d'emploi.

Le recrutement a été effectué au sein d'un organisme communautaire de l'Outaouais québécois qui offre du soutien aux jeunes LGB. Les participants ont complété le questionnaire anonyme sur place, dans un local permettant de protéger leur confidentialité et ils l'ont ensuite remis, sous enveloppe scellée, dans un boîtier verrouillé et sécurisé.

\section{Instruments}

Les participants ont complété un livret regroupant les questionnaires qui ont permis de mesurer le soutien social, le rapport à l'orientation sexuelle, la détresse psychologique, le bien-être et le fonctionnement scolaire. Le soutien de la famille (12 items, $\alpha=0,93)$ et des amis (13 items, $\alpha=0,90$ ) a été mesuré au moyen de l'Échelle de soutien social (adaptée d'après Boies, Cooper \& Osborne, 2004), tandis que le rapport à l'orientation sexuelle a été évalué grâce aux sous-échelles de confusion ( 5 items, $\alpha=0,79$ ), de besoin d'acceptation ( 6 items, $\alpha=0,73$ ) et d'homophobie internalisée ( 8 items, $\alpha=0,87$ ) de l'Échelle de l'identité gaie et lesbienne (adaptée d'après Mohr \& Fassinger, 2000). Les mesures de détresse psychologique incluaient l'Échelle d'anxiété de Costello-Comrey (1967; 9 items, $\alpha=0,92$ ), l'Auto-évaluation brève de dépression (Hakstian \& McLean, 1989, 4 items, $\alpha=0,83$ ) et la sous-échelle de l'inquiétude de subir de la violence homophobe (4 items, $\alpha=0,79)$ extraite de l'Échelle des événements stressants (adaptée d'après Polders, Nel, Kruger, \& Wells, 2006). Les instruments qui ont servi à évaluer le bien-être psychologique étaient l'Affectomètre (Kammann \& Flett, 1983; 8 items, $\alpha=0,86$ ), l'Échelle de satisfaction envers la vie (Diener, Emmons, Larsen, \& Griffin, 1985; 5 items, $\alpha=0,84$ ), l'Échelle de locus de contrôle (adapté d'après Levenson, 1981; 4 items, $\alpha=0,71$ ) et l'Échelle d'estime de soi (Rosenberg, 1965, 10 items, $\alpha=0,94$ ). Les mesures de fonctionnement 
scolaire comprennent l'Inventaire des comportements interpersonnels (d'après Pelletier \& Otis, 2002; 4 items, $\alpha=0,80$ ) qui a permis d'évaluer le soutien des enseignants, l'Échelle de motivation en éducation (Vallerand Blais, Brière, \& Pelletier, 1989; 24 items, a global $=0,95$ ) et la sous-échelle de 3 items $(\alpha=0,81)$ mesurant la violence homophobe à l'école de l'Échelle des événements stressants (adapté d'après Polders et al., 2006).

\section{RÉSULTATS}

\section{Statistiques descriptives}

La moyenne et l'écart-type des variables étudiées sont présentés au Tableau 1. Le niveau moyen de soutien de la famille était modérément élevé tandis que celui des amis était très élevé. En ce qui concerne les attitudes reliées à l'orientation sexuelle, les participants ont rapporté un niveau faible de confusion ainsi qu'un niveau modérément faible de besoin d'acceptation et d'homophobie internalisée. L'amplitude moyenne des variables positives de bien-être psychologique (émotions positives, locus de contrôle, estime de soi et satisfaction envers la vie) était modérément élevée. En contraste, la valeur moyenne des variables négatives de bienêtre psychologique (anxiété, dépression et inquiétudes reliées à la violence homophobe) était modérément faible. De plus, en ce qui concerne les variables scolaires, le niveau de soutien des enseignants était modérément élevé, la motivation scolaire était modérée et la violence homophobe rapportée était faible.

Par ailleurs, un problème de normalité de la distribution du soutien des amis a été identifié (kurtose $=7,91$, asymétrie $=-2,38$ ). Cette distribution présente un effet de plafond parce que les participants ont rapporté un niveau élevé de soutien des amis. Afin de gérer ce problème, cette variable a été transformée (en générant l'inverse de la distribution réfléchie) aux fins des analyses subséquentes, ce qui a normalisé sa distribution (kurtose $=-0,87$; asymétrie $=0,01$ ).

Associations entre le soutien social, le rapport à l'orientation sexuelle, la détresse et le bien-être psychologiques

Des corrélations ont été évaluées entre le soutien de la famille et des amis, d'une part, et les variables indicatives du rapport à l'orientation sexuelle, de la détresse et du bien-être psychologiques, d'autre part. Les résultats sont rapportés au Tableau 2.

Le soutien de la famille est négativement associé aux trois variables qui suggèrent un rapport conflictuel à l'orientation sexuelle (la confusion, le besoin d'acceptation et l'homophobie internalisée), négativement associé à 
Soutien social, détresse et bien-être chez les jeunes LGB

Tableau 1

Statistiques descriptives

\begin{tabular}{lcc}
\hline & Moyenne & Écart-type \\
\hline $\begin{array}{l}\text { Variables sociales } \\
\text { Soutien famille }\end{array}$ & 4,82 & 1,44 \\
$\quad$ Soutien amis & 6,26 & 0,83 \\
Rapport à l'orientation sexuelle & & \\
$\quad$ Confusion & 1,57 & 0,81 \\
$\quad$ Besoin d'acceptation & 2,93 & 1,28 \\
$\quad$ Homophobie internalisée & 2,35 & 1,37 \\
Détresse psychologique & & \\
$\quad$ Symptômes anxieux & 3,67 & 1,56 \\
$\quad$ Symptômes dépressifs & 3,22 & 1,64 \\
$\quad$ Inquiétude liée à la violence homophobe & 1,52 & 0,60 \\
Bien-être psychologique & & 1,20 \\
$\quad$ Émotions positives & 4,77 & 1,33 \\
$\quad$ Satisfaction envers la vie & 4,97 & 1,28 \\
$\quad$ Locus de contrôle & 5,01 & 1,45 \\
$\quad$ Estime de soi & 5,24 & 1,26 \\
Fonctionnement scolaire & & 1,79 \\
$\quad$ Soutien des professeurs & 5,28 & 0,69 \\
$\quad$ Motivation scolaire & 1,81 & \\
Violence homophobe à l'école & 1,67 & \\
\hline
\end{tabular}

Note. L'étendue théorique est de 1 à 7 pour toutes les variables sauf pour l'inquiétude liée à la violence homophobe ( 1 à 4$)$, la violence homophobe à l'école (1 à 4 ) et la motivation scolaire $(-6$ à +6$)$.

\section{Tableau 2}

Associations entre le soutien social, le rapport à l'orientation sexuelle, la détresse et le bien-être psychologiques

\begin{tabular}{|c|c|c|c|c|}
\hline & \multicolumn{2}{|c|}{ Soutien de la famille } & \multicolumn{2}{|c|}{ Soutien des amis } \\
\hline & $\begin{array}{c}\text { Corrélation } \\
\text { d'ordre } 0\end{array}$ & 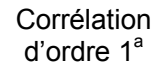 & $\begin{array}{c}\text { Corrélation } \\
\text { d'ordre } 0\end{array}$ & 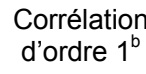 \\
\hline \multicolumn{5}{|l|}{ Rapport à l'orientation sexuelle } \\
\hline Confusion & $-0,41^{*}$ & $-0,34^{*}$ & $-0,28$ & $-0,08$ \\
\hline Besoin d'acceptation & $-0,67^{* * *}$ & $-0,70^{* * *}$ & $-0,20$ & 0,30 \\
\hline Homophobie internalisée & $-0,41^{*}$ & $-0,40^{*}$ & $-0,16$ & 0,08 \\
\hline
\end{tabular}


RQP, 37(1)

Tableau 2

Associations entre le soutien social, le rapport à l'orientation sexuelle, la détresse et le bien-être psychologiques (suite)

\begin{tabular}{|c|c|c|c|c|}
\hline & \multicolumn{2}{|c|}{ Soutien de la famille } & \multicolumn{2}{|c|}{ Soutien des amis } \\
\hline & $\begin{array}{l}\text { Corrélation } \\
\text { d'ordre } 0\end{array}$ & 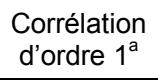 & $\begin{array}{l}\text { Corrélation } \\
\text { d'ordre } 0\end{array}$ & $\begin{array}{l}\text { Corrélation } \\
{\text { d'ordre } 1^{\mathrm{b}}}\end{array}$ \\
\hline \multicolumn{5}{|l|}{ Détresse psychologique } \\
\hline Symptômes anxieux & $-0,43^{*}$ & $-0,45^{\star *}$ & $-0,14$ & 0,16 \\
\hline Symptômes dépressifs & $-0,55^{\star *}$ & $-0,50^{* *}$ & $-0,30$ & $-0,02$ \\
\hline $\begin{array}{l}\text { Inquiétude liée à la violence } \\
\text { homophobe }\end{array}$ & $-0,60^{* * *}$ & $-0,50^{* *}$ & $-0,44^{* *}$ & $-0,19$ \\
\hline \multicolumn{5}{|l|}{ Bien-être psychologique } \\
\hline Émotions positives & $0,56^{\star * *}$ & $0,47^{* *}$ & $0,44^{* *}$ & 0,17 \\
\hline Satisfaction envers la vie & $0,52^{\star *}$ & $0,40^{*}$ & $0,45^{\star *}$ & 0,24 \\
\hline Locus de contrôle & $0,63^{* * *}$ & $0,52^{* *}$ & $0,52^{* *}$ & 0,27 \\
\hline Estime de soi & $0,60^{* * *}$ & $0,50^{* *}$ & $0,44^{* *}$ & 0,21 \\
\hline
\end{tabular}

Notes. $a=$ corrélations partielles où la covariable dont l'influence est contrôlée est le soutien des amis; $b=$ corrélations partielles où la covariable dont l'influence est contrôlée est le soutien de la famille; ${ }^{*} p<.05,{ }^{* *} p<.01,{ }^{* * *} p<.001$, test unilatéral.

la détresse psychologique (symptômes anxieux et dépressifs, inquiétude liée à la violence homophobe) et positivement associé au bien-être (émotions positives, satisfaction envers la vie, locus de contrôle et estime de soi). Ces corrélations sont statistiquement significatives et de taille importante. Tel qu'indiqué dans la seconde colonne du Tableau 2, ces associations demeurent significatives et de taille élevée lorsqu'elles sont évaluées en extrayant l'influence du soutien des amis.

Le soutien des amis est associé négativement à l'inquiétude liée à la violence homophobe et positivement à toutes les variables de bien-être psychologique (émotions positives, satisfaction envers la vie, locus de contrôle et estime de soi). Ces corrélations sont élevées et statistiquement significatives. Les associations entre le soutien des amis, d'une part, et la confusion, le besoin d'acceptation, l'homophobie internalisée, les symptômes anxieux et dépressifs, d'autre part, ne sont pas significatives. Toutes les associations significatives reliées au soutien des amis disparaissent lorsque l'influence du soutien des parents est contrôlée (voir colonne 4, Tableau 2). 
Associations entre le rapport à l'orientation sexuelle, la détresse et le bienêtre

Des corrélations ont été évaluées entre les composantes du rapport à l'orientation sexuelle, d'une part, et la détresse et le bien-être psychologiques, d'autre part (voir Tableau 3). Dans l'ensemble, le rapport conflictuel à l'orientation sexuelle (confusion, besoin d'acceptation, homophobie internalisée) est associé positivement à la détresse psychologique (symptômes anxieux et dépressifs, inquiétudes liées à la violence homophobe) et négativement au bien-être (émotions positives, satisfaction envers la vie, locus de contrôle et estime de soi). Ces corrélations sont statistiquement significatives et leur amplitude est substantielle. Seule la corrélation entre l'homophobie internalisée et l'estime de soi était non significative.

\section{Associations entre les facteurs de fonctionnement scolaire}

Le soutien des enseignants est positivement associé à la motivation scolaire $(r=0,56, p<0,01)$ et négativement associé à la violence homophobe verbale et physique vécue à l'école $(r=-0,47, p<0,05)$. De plus, la violence homophobe vécue à l'école est négativement associée à la motivation scolaire $(r=-0,53, p<0,01)$. Ces associations sont de taille élevée.

Tableau 3

Associations entre le rapport à l'orientation sexuelle, le bien-être et la détresse psychologique

\begin{tabular}{llcc}
\hline & \multicolumn{2}{c}{ Rapport à l'orientation sexuelle } \\
\cline { 2 - 4 } & Confusion & $\begin{array}{c}\text { Besoin } \\
\text { d'acceptation }\end{array}$ & $\begin{array}{c}\text { Homophobie } \\
\text { internalisée }\end{array}$ \\
\hline $\begin{array}{l}\text { Détresse psychologique } \\
\text { Symptômes anxieux }\end{array}$ & $0,48^{* *}$ & $0,54^{* *}$ & $0,40^{*}$ \\
$\quad$ Symptômes dépressifs & $0,53^{* *}$ & $0,49^{* *}$ & $0,46^{* *}$ \\
$\quad$ Inquiétude liée à la violence & $0,44^{* *}$ & $0,68^{* *}$ & $0,60^{* * *}$ \\
homophobe & & & \\
Bien-être psychologique & & & $-0,44^{* *}$ \\
Émotions positives & $-0,44^{* *}$ & $-0,47^{* *}$ & $-0,34^{*}$ \\
Satisfaction envers la vie & $-0,43^{* *}$ & $-0,35^{*}$ & $-0,33^{*}$ \\
Locus de contrôle & $-0,45^{* *}$ & $-0,45^{* *}$ & $-0,31$ \\
Estime de soi & $-0,43^{* *}$ & $-0,40^{*}$ & \\
\hline
\end{tabular}

Notes. ${ }^{*} p<.05,{ }^{* *} p<.01,{ }^{* *} p<.001$, test unilatéral. 


\section{DISCUSSION}

Le présent projet visait à examiner le soutien social de la famille et des amis, le rapport à l'orientation sexuelle, la détresse psychologique, le bienêtre et le fonctionnement scolaire de jeunes LGB québécois. L'hypothèse (1) prévoyait que le soutien des parents et des amis serait négativement associé à un rapport conflictuel à l'orientation sexuelle (confusion, besoin d'acceptation, homophobie internalisée). Cette hypothèse a été partiellement corroborée. Tandis que le soutien de la famille était significativement et négativement associé aux variables reliées au rapport conflictuel à l'orientation sexuelle, le soutien des amis n'a pas fait montre, pour cet échantillon, de corrélations significatives. Ceci suggère donc que plus le soutien de la famille est élevé, moins les jeunes LGB se sentent confus par rapport à leur orientation sexuelle, moins ils ressentent le besoin que leur orientation sexuelle soit approuvée socialement et moins ils souffrent d'homophobie internalisée. Ces résultats vont dans le même sens que ceux de Bregman et ses collaborateurs (2013) et ceux de Floyd et ses collaborateurs (1999), indiquant que la perception du soutien des parents pourrait favoriser le processus de consolidation de l'identité sexuelle des jeunes LGB. Shilo et Savaya (2011) ont également trouvé que le soutien de la famille était plus fortement relié à l'acceptation de soi en tant que LGB, alors que Feinstein et ses collaborateurs (2014) ont obtenu des résultats indiquant que, plus l'attitude des parents était positive envers l'orientation sexuelle de leur jeune, moins celui-ci présentait d'homophobie internalisée.

En raison de la taille réduite de l'échantillon et parce que les participants bénéficiaient d'un soutien uniformément fort de la part de leurs amis, il est difficile d'interpréter avec clarté les associations non significatives entre cette variable et le rapport à l'orientation sexuelle. Dans un premier temps, il est possible que ces résultats reflètent la réalité. Un patron clair d'associations négatives d'amplitude acceptable (non statistiquement significatives à cause d'un manque de puissance statistique) émerge entre le soutien des amis et le rapport conflictuel à l'orientation sexuelle. Ces associations disparaissent lorsque l'influence du soutien de la famille est contrôlée. Pris ensemble, ces résultats pourraient indiquer que, lorsque la famille est une source de soutien, elle joue un rôle de protection qui amoindrit l'influence négative des pairs. Dans un deuxième temps, il est possible que l'amplitude élevée et la dispersion réduite des scores représentant le soutien des amis aient entravé l'émergence d'associations impliquant cette variable. Au plan statistique, la corrélation est obtenue en standardisant la covariance. Pour obtenir un degré suffisant de covariance, les variables impliquées doivent d'abord individuellement présenter de la variabilité. Autrement dit, la corrélation évalue si deux variables changent de façon concomitante (dans le même sens ou dans des directions opposées). Si l'une de ces variables change 
peu, ses scores se prêtent mal à l'obtention de corrélations. C'est le cas du soutien des amis dans cette étude qui présente un effet de plafond (moyenne élevée et écart-type réduit). Cet effet de plafond résulte probablement du contexte dans lequel le recrutement a été effectué, soit par l'entremise d'un organisme offrant du soutien aux jeunes LGB. Pour remédier à ce problème, il faudrait, lors d'études futures, évaluer à nouveau les associations impliquant le soutien des amis, auprès d'échantillons où cette variable fluctue davantage.

L'hypothèse (2) postulait que le soutien des parents et des amis serait négativement associé à la détresse psychologique et positivement associé au bien-être. Cette hypothèse a été corroborée pour le soutien des parents et non confirmée pour le soutien des amis. En effet, d'une part, suite aux corrélations partielles effectuées, le soutien de la famille était significativement et négativement associé aux symptômes anxieux, aux symptômes dépressifs et à l'inquiétude liée à la violence homophobe et positivement associé aux émotions positives, à la satisfaction envers la vie, au locus de contrôle et à l'estime de soi, même lorsque l'influence des amis était contrôlée. Cette influence bénéfique du soutien familial est congruente avec la documentation importante à ce sujet (Anderson, 1998; Bregman et al., 2013; Floyd et al., 1999; Needham \& Austin, 2010; Shilo \& Savaya, 2011; Teasdale \& Bradley-Engen, 2010). II est utile de souligner qu'il est intéressant de constater que ces effets se retrouvent aussi dans l'échantillon de jeunes LGB québécois de la présente étude, car il s'agit d'une population qui fait l'objet d'une documentation en émergence.

D'autre part, les associations prévues entre le soutien des amis, la détresse et le bien-être n'ont pas été corroborées pour cet échantillon. Ces résultats ne sont pas congruents avec ceux des auteurs (p. ex., Mustanski et al., 2011) qui considèrent que le soutien des pairs est celui qui diminue le plus fortement la détresse psychologique. II n'y a toutefois pas de consensus dans la littérature concernant l'impact du soutien des pairs sur les LGB puisque d'autres auteurs révèlent que les pairs peuvent avoir un effet négatif sur la santé mentale de ces jeunes et que ceux-ci seraient moins portés à ressentir leur support social (Teasdale \& Bradley-Engen, 2010).

Il est d'abord important de mentionner qu'en raison du niveau élevé et de la variabilité réduite des scores du soutien des amis, les résultats de l'hypothèse (2) reliés à cette variable dans le cadre de la présente étude sont limités par les mêmes considérations que celles qui ont été évoquées au sujet de l'hypothèse (1). Par ailleurs, lors d'études futures, il serait également fructueux de mesurer de façon conjointe le soutien des amis et l'influence des pairs de façon à départager leur influence. Cette remarque est relative à l'ambiguïté des résultats reliés à ces variables dans la 
présente étude de même que dans la documentation. II est plausible de concevoir que le soutien des amis soit susceptible d'être plus positif que l'influence des pairs plus éloignés (qui pourrait comporter des attitudes plus néfastes et destructives). II serait également utile de prendre en considération l'orientation sexuelle des amis ainsi que la réaction des amis quant à l'orientation sexuelle des jeunes LGB. II est permis d'espérer que les amis soient une source de soutien et de réconfort qui transcende l'orientation sexuelle dans certains cas. II est toutefois aussi tristement probable que les expériences d'hostilité et de rejet soient plus douloureuses lorsqu'elles proviennent de personnes avec qui un lien affectif d'amitié est déjà établi que lorsqu'elles impliquent des connaissances plus distales ou des étrangers.

En somme, de nombreux facteurs sont susceptibles de nuancer et de complexifier les associations entre le rôle des amis et des pairs et le bienêtre ou la détresse chez les jeunes LGB. II serait très important d'examiner ces questions plus à fond lors d'études futures, car une meilleure compréhension de ces facteurs reliés au réseau social des jeunes LGB est essentielle pour pouvoir mieux intervenir auprès de cette population. II est ainsi important de souligner qu'il faut éviter de conclure, sur la base des résultats de cette étude, que les interventions doivent seulement être effectuées auprès de la famille et non pas auprès des amis. En effet, le soutien de la famille gagne à être évalué avant d'intervenir en ce sens, de même que les difficultés possibles que les jeunes LGB auraient pu vivre au sein de leurs relations familiales.

L'hypothèse (3) prédisait que le rapport conflictuel à l'orientation sexuelle serait positivement associé à la détresse psychologique et négativement au bien-être. La confusion de l'identité, le besoin d'acceptation et l'homophobie internalisée étaient effectivement reliés positivement aux symptômes anxieux et dépressifs ainsi qu'à l'inquiétude par rapport à la violence homophobe. Les variables dénotant un rapport difficile à l'orientation sexuelle étaient, de plus, négativement associées aux émotions positives, à la satisfaction envers la vie, au locus de contrôle et à l'estime de soi. Ces résultats sont congruents avec ceux de la documentation importante sur l'homophobie internalisée qui associe cette variable à un vaste éventail de troubles psychopathologiques (Benibgui, 2011; Feinstein et al., 2014; Newcomb \& Mustanski, 2010).

L'hypothèse (4) qui stipulait que le soutien des enseignants serait positivement associé à la motivation scolaire et négativement associé à la violence homophobe verbale et physique vécue à l'école a été confortée dans cette étude. Le présent projet révèle donc que, plus les professeurs offrent un soutien aux élèves LGB, plus ces derniers ont une motivation scolaire élevée et moins ils vivent de violence verbale ou physique à 
l'école. Ces résultats vont dans le même sens que ceux de Murdock et Bolch (2005), révélant une association négative entre le support des professeurs et la violence homophobe, les problèmes de discipline ainsi que sur l'exclusion scolaire. Bien que la motivation scolaire n'ait pas été évaluée dans cette étude, ces auteurs ont aussi observé des associations positives entre le soutien des professeurs, le rendement scolaire chez les jeunes LGB et que le sentiment d'appartenance à l'école.

L'hypothèse (5) qui prédisait que la violence homophobe vécue à l'école serait négativement associée à la motivation scolaire a été corroborée. Dans cet échantillon, lorsque les jeunes vivaient de la violence liée à l'orientation sexuelle dans leur milieu scolaire, la motivation scolaire était significativement plus faible. Chez les LGB, il a été révélé précédemment que les abus verbaux ou physiques par les pairs sont une source de stress supplémentaire qui peut être reliée à des conséquences néfastes, telles que des problèmes psychologiques (D'Augelli et al., 2005). D'autres études ont rapporté que les LGB avaient une connexion plus faible à leur établissement scolaire (Saewyc et al., 2009) et que leur taux d'absentéisme était plus élevé quand ils vivaient de la discrimination (Martin \& Beaulieu, 2002). Toutefois, à notre connaissance, aucune étude antérieure n'a porté directement sur la motivation scolaire des jeunes LGB du Québec ou d'ailleurs. II s'agit donc d'une contribution novatrice du présent projet.

Il est important de reconnaître que la présente étude comporte des limites méthodologiques. En effet, cette étude est de nature exploratoire et la taille de l'échantillon est réduite. II serait nécessaire de chercher à répliquer les résultats obtenus ici dans le cadre d'études impliquant des échantillons plus volumineux.

L'orientation sexuelle était uniquement basée sur l'auto-identification du participant, ce qui constitue une limite additionnelle, car plusieurs personnes qui s'identifient comme étant hétérosexuelles rapportent également des comportements ou des fantaisies sexuels avec des personnes du même sexe (Laumann, Gagnon, Michael, \& Michaels, 1994; Zhao et al., 2010). L'identification de l'orientation sexuelle s'avère une mesure complexe à évaluer puisqu'elle peut inclure un ou des éléments parmi l'auto-identification des participants, leurs comportements sexuels, leurs attirances sexuelles, leurs préférences émotionnelles, etc. - dans le passé, le présent et dans l'idéal des participants (Weinrich \& Klein, 2002).

Par ailleurs, le devis de la présente étude est de type corrélationnel. II n'est donc pas possible de déterminer avec exactitude la direction des relations entre les variables étudiées et de départager clairement les 
causes antécédentes de leurs conséquences. La réalisation d'études longitudinales est requise pour approfondir cette question.

Le soutien de la famille a été fortement relié à la réduction de la détresse psychologique (Shilo \& Savaya, 2011) et au bien-être des LGB (Floyd et al., 1999; Needham \& Austin, 2010). La santé mentale est particulièrement à risque chez les jeunes LGB qui sont plus susceptibles que leurs pairs hétérosexuels de développer un trouble anxieux ou une dépression majeure (King et al., 2008), des abus de substances (Birkett et al., 2009; Klitzman, Greenberg, Pollack, \& Dolezal, 2002), des troubles des conduites (Fergusson et al., 1999), des troubles alimentaires et d'insatisfaction corporelle (Hospers \& Jansen, 2005; Russell \& Keel, 2002), des comportements d'automutilation (Rivers, 2000) et des comportements suicidaires au cours de leur vie (King et al., 2008).

II est permis d'espérer que la présente étude contribuera utilement à amorcer des projets plus ambitieux qui permettront de mieux comprendre les sources de détresse et de bien-être psychologiques des jeunes LGB québécois afin d'isoler les facteurs de risque qui fragilisent cette population ainsi que les facteurs de protection qui favorisent la résilience.

\section{RÉFÉRENCES}

Ainsworth, M. D., Blehar, M. C., Waters, E., \& Wall, S. (1978). Patterns of attachment: A theoretical review of the infant-mother relationship. Child Development, 40, 969-1025.

Alivernini, F., \& Lucidi, F. (2011). Relationship between social context, self-efficacy, motivation, academic achievement, and intention to drop out of high school: A longitudinal study. The Journal of Educational Research, 104(4), 241-252.

Anderson, A. L. (1998). Strengths of gay male youth: An untold story. Child and adolescent social work journal, 15(1), 55-71.

Anhalt, K., \& Morris, T. L. (1998). Developmental and adjustment issues of gay, lesbian, and bisexual adolescents: A review of the empirical literature. Clinical Child and Family Psychology Review, 1(4), 215-230.

Assemblée nationale Québec. (2012). Projet de loi $n^{\circ} 56$ : Loi visant à prévenir et à combattre l'intimidation et la violence à l'école. Consulté de http://www.assnat.qc.ca/fr/travauxparlementaires/projets-loi/projet-loi-56-39-2.html

Balsam, K. F., \& Mohr, J. J. (2007). Adaptation to sexual orientation stigma: A comparison of bisexual and lesbian/gay adults. Journal of Counseling Psychology, 54(3), 306-319.

Benibgui, M. (2011). Mental health challenges and resilience in lesbian, gay, and bisexual young adults: Biological and psychological internalization of minority stress and victimization (thèse de doctorat non publiée). Université Concordia, Canada.

Berghe, W. V., Dewaele, A., Cox, N., \& Vincke, J. (2010). Minority-specific determinants of mental well-being among lesbian, gay, and bisexual youth. Journal of Applied Social Psychology, 40(1), 153-166.

Birkett, M., Espelage, D. L., \& Kœnig, B. (2009). LGB and Questioning students in schools: The moderating effects of homophobic bullying and school climate on negative outcomes. Journal of Youth Adolescence, 38(7), 989-1000.

Boies, S. C., Cooper, A., \& Osborne, C. S. (2004). Variations in Internet-related problems and psychosocial functioning in online sexual activities: Implications for social and sexual development of young adults. CyberPsychology \& Behavior, 7(2), 207-230. 
Bos, H. M. W., Sandfort, T. G. M., Bruyn, E. H., \& Hakvoort, E. M. (2008). Same-sex attraction, social relationships, psychosocial functioning, and school performance in early adolescence. Developmental Psychology, 44(1), 59-68.

Bostwick, W. B., Boyd, C. J., Hughes, T. L., \& McCabe, S. E. (2010). Dimensions of sexual orientation and the prevalence of mood and anxiety disorders in the United States. American Journal of Public Health, 100(3), 468-475.

Bowlby, J. (1969). Attachment and loss: Vol. 1. Attachment. New York, NY: Basic Books.

Bowlby, J. (1973). Attachment and loss: Vol. 2. Separation. New York, NY: Basic Books.

Bregman, H. R., Malik, N. M., Page, M. J. L., Makynen, E., \& Lindahl, K. M. (2013). Identity profiles in lesbian, gay, and bisexual youth: The role of family influences. Journal of Youth and Adolescence, 42(3), 417-430.

Bricker, M. E. (2009). The role of emotional approach coping and internalized homophobia in perceived stress, depression, and meaning of life among gay men and lesbians. The Sciences and Engineering, 69(9-B), 5768.

Carter II, L. W., Mollen, D., \& Smith, N. G. (2014). Locus of control, minority stress, and psychological distress among lesbian, gay, and bisexual individuals. Journal of Counseling Psychology, 61(1), 169-175.

Chamberland, L., Émond, G., Julien, D., Otis, J., \& Ryan, B. (2010). L'impact de l'homophobie et de la violence homophobe sur la persévérance et la réussite scolaires: Rapport de recherche. En ligne: http://www.fqrsc.gouv.qc.ca/fr/rechercheexpertise/projets/rapports recherche.php\#PRS2006

Costello, C. G., \& Comrey, A. L. (1967). Scales for measuring depression and anxiety. The Journal of Psychology, 66(2), 303-313.

D’Amico, É., Julien, D., Tremblay, N., \& Chartrand, É. (2012). Réactions des parents à la suite du dévoilement de l'orientation sexuelle de leur enfant gai, lesbienne ou bisexual. Nouvelles pratiques sociales, 24(2), 120-139.

D’Augelli, A. R., Grossman, A. H., Salter, N. P., Vasey, J. J., Starks, M. T., \& Sinclair, K. O. (2005). Predicting the suicide attempts of lesbian, gay, and bisexual youth. Suicide and Life-Threatening Behavior, 35(6), 646-660.

D’Augelli, A. R., \& Hershberger, S. L. (1993). Lesbian, gay, and bisexual youth in community settings: Personal challenges and mental health problems. American Journal of Community Psychology, 21(4), 421-448.

D’Augelli, A. R., Pilkington, N. W., \& Hershberger, S. L. (2002). Incidence and mental health impact of sexual orientation victimization of lesbian, gay, and bisexual youths in high school. School Psychology Quarterly, 17(2), 148-167.

Diener, E., Emmons, R. A., Larsen, R. J., \& Griffin, S. (1985). The satisfaction with life scale. Journal of Personality Assessment, 49(1), 71-75.

Eliason, M. J. (1996). Identity formation for lesbian, bisexual, and gay persons: Beyond a "minoritizing" view. Journal of Homosexuality, 30(3), 31-58.

Feinstein, B. A., Wadsworth, L. P., Davila, J., \& Goldfried, M. R. (2014). Do parental acceptance and family support moderate associations between dimensions of minority stress and depressive symptoms among lesbians and gay men? Professional Psychology: Research and Practice, 45(4), 239-246.

Fergusson, D. M., Horwood, L. J., \& Beautrais, A. L. (1999). Is sexual orientation related to mental health problems and suicidality in young people? Archives of General Psychiatry, 56(10), 876-880.

Floyd, F. J., Stein, T. S., Harter, K. S. M., Allison, A., \& Nye, C. L. (1999). Gay, lesbian, and bisexual youths: Separation-individuation, parental attitudes, identity consolidation, and well-being. Journal of Youth and Adolescence, 28(6), 719-739.

Goodenow, C., Szalacha, L., \& Westheimer, K. (2006). School support groups, other school factors, and the safety of sexual minority adolescents. Psychology in the Schools, 43(5), 573-589.

Green-Demers, I., Mageau, G. A., \& Pelletier, D. (2013). L'impact du style de leadership des enseignants et des valeurs des élèves sur la croissance personnelle, la motivation, la réussite et la persévérance scolaires. Rapport de recherche intégral. Programme des actions concertées sur la réussite et la persévérance scolaires, Fonds de recherche Société et culture Québec. Québec, Canada. 
Guay, F., Ratelle, C. F., \& Chanal, J. (2008). Optimal learning in optimal contexts: The role of self-determination in education. Psychologie canadienne, 49(3), 233-240.

Hakstian, A. R., \& McLean, P. D. (1989). Brief screen for depression. Psychological Assessment: Journal of Consulting and Clinical Psychology, 1(2), 139-141.

Harris, M. B. (1997). School experiences of gay and lesbian youth: The invisible minority. Binghamton, NY: Harworth Press.

Hatzenbuehler, M. L. (2011). The social environment and suicide attempts in lesbian, gay, and bisexual youth. Pediatrics, 127(5), 896-903.

Hershberger, S. L., \& D'Augelli, A. R. (1995). The impact of victimization on the mental health and suicidality of lesbian, gay, and bisexual youths. Developmental Psychology, 31(1), 65-74.

Hospers, H. J., \& Jansen, A. (2005). Why homosexuality is a risk factor for eating disorders in males. Journal of Social and Clinical Psychology, 24(8), 1188-1201.

Igartua, K. J., Gill, K., \& Montoro, R. (2003). Internalized homophobia: A factor in depression, anxiety, and suicide in the gay and lesbian population. Revue canadienne de santé mentale communautaire, 22(2), 15-30.

Igartua, K. J., Thombs, B. D., Burgos, G., \& Montoro, R. (2009). Concordance and discrepancy in sexual identity, attraction, and behavior among adolescents. Journal of Adolescent Health, 45(6), 602-608.

Infurna, F. J., Gerstorf, D., Ram, N. Schupp, J., \& Wagner, G. G. (2011). Long-term antecedents and outcomes of perceived control. Psychology and aging, 26(3), 559-575.

Julien, D., \& Chartrand, É. (2005). Recension des écrits sur la santé des personnes gaies, lesbiennes et bisexuelles. Psychologie Canadienne, 46(4), 235-250.

Kammann, R., \& Flett, R. (1983). Affectometer 2: A scale to measure current level of general happiness. Australian Journal of Psychology, 35(2), 259-265.

King, L. A., \& Smith, N. G. (2004). Gay and straight possible selves: Goals, identity, subjective well-being, and personality development. Journal of Personality, 72(5), 967-994.

King, M., Semlyen, J., Tai, S. S., Killaspy, H., Osborn, D., Popelyuk, D. et al. (2008). A systematic review of mental disorder, suicide, and deliberate self-harm in lesbian, gay and bisexual people. BMC Psychiatry, 8, 1-17.

Klitzman, R. L., Greenberg, J. D., Pollack, L. M., \& Dolezal, C. (2002). MDMA ("ecstasy") use, and its association with high risk behaviors, mental health, and other factors among gay/bisexuals men in New York City. Drug and Alcohol Dependence, 66(2), 115-125.

Knight, L. F. M., \& Hope, D. A.(2012). Correlates of same-sex attractions and behaviors among self-identified heterosexual university students. Archives of Sexual Behavior, 41(5), 1199-1208.

Langis, P., Germain, B., Dallaire, Y., Normandeau, D., \& Ross, M. (2015). La sexualité humaine ( $2^{\mathrm{e}}$ éd.). Louvain-la-Neuve : De Boeck Supérieur.

Laumann, E. O., Gagnon, J. H., Michael, R. T., \& Michaels, S. (1994). The social organization of sexuality: Sexual practices in the United States. Chicago, IL : University of Chicago Press.

Lavoie, K., \& Côté, I. (2014). L'expérience des parents d'un enfant d'orientation homosexuelle: savoirs issus des recherches et perspectives d'intervention. Service social, 60(1), 15-33.

Lazarus, R. S. (1990). Stress, coping and illness. In H. S. Friedman (Éd.), Personality and disease (p. 97-120). New York, NY : John Wiley \& Sons.

Lazarus, R. S., \& Folkman, S. (1984). Stress, appraisal and coping. New York, NY : Springer Publishing.

Lee, R. M., Dean, B. L., \& Jung, K.-R. (2008). Social connectedness, extraversion, and subjective well-being: Testing a mediation model. Personality and Individual Differences, 45(5), 414-419.

Levenson, H. (1981). Differentiating among internality, powerful others, and chance. In H.M. Lefcourt (Éd.), Research with the locus of control construct. Vol.1. Assessment methods (p. 15-63). New York, NY : Academic Press.

Liu, R. T., \& Mustanski, B. (2012). Suicidal ideation and self-harm in lesbian, gay, bisexual, and transgender youth. American Journal of Preventive Medicine, 42(3), 221-228. 
Marshal, M. P., Dietz, L. J., Friedman, M. S., Stall, R., Smith, H. A., \& McGinley, J. (2011). Suicidality and depression disparities between sexual minority and heterosexual youth: $A$ meta-analytic review. Journal of Adolescent Health, 49(2), 115-123.

Martin, D., \& Beaulieu, A. (2002). Besoins des jeunes homosexuelles et homosexuels et interventions en milieu scolaire pour contrer l'homophobie. Commission scolaire de Montréal, Services des ressources éducatives.

Mikulincer, M., \& Shaver, P. R. (2007). Attachment in adulthood: structure, dynamics, and change. New York, NY: Guilford Press.

Mohr, J., \& Fassinger, R. (2000). Measuring dimensions of lesbian and gay male experience. Measurement and Evaluation in Counseling and Development, 33(2), 66-90.

Murdock, T. B., \& Bolch, M. B. (2005). Risk and protective factors for poor school adjustment in lesbian, gay, and bisexual (LGB) high school youth: Variable and person-centered analyses. Psychology in the Schools, 42(2), 159-172.

Mustanski, B., Newcomb, M. E., \& Garofalo, R. (2011). Mental health of lesbian, gay, and bisexual youths: A developmental resiliency perspective. Journal of Gay \& Lesbian Social Services, 23(2), 204-225.

Needham, B. L., \& Austin, E. L. (2010). Sexual orientation, parental support, and health during the transition to young adulthood. Journal of Youth and Adolescence, 39(10), 1189-1198.

Newcomb, M. E., \& Mustanski, B. (2010). Internalized homophobia and internalizing mental health problems: A meta-analytic review. Clinical Psychology Review, 30(8), 1019-1029.

Ortiz-Hernández, L. (2005). Influencia de la opresión internalizada sobre la salud mental de bisexuales, lesbianas y homosexuales de la ciudad de México. Salud Mental, 28(4), 4965.

Pelletier, L. G., \& Otis, N. (2002). Construction et validation de l'échelle des comportements interpersonnels. Manuscrit inédit.

Peter, T., Taylor, C., \& Chamberland, L. (2015). A queer day in Canada: Examining Canadian high school students' experiences with school-based homophobia in two large-scale studies. Journal of Homosexuality, 62(2), 186-206.

Petit, M.-P., Chamberland, L., Richard, G., \& Chevrier, M. (2011). Jeunes de minorités sexuelles victimes d'homophobie en milieu scolaire: quels facteurs de protection? Revue canadienne de santé mentale communautaire, 30(2), 13-29.

Polders, L. A., Nel, J. A., Kruger, P., \& Wells, M. (2006). Factors affecting vulnerability to depression among gay men and lesbian women in Gauteng, South Africa. South African Journal of Psychology, 38(4), 673-687.

Potoczniak, D. J., Aldea, M. A., \& DeBlaere, C. (2007). Ego identity, social anxiety, social support, and self-concealment in lesbian, gay, and bisexual individuals. Journal of Conseling Psychology, 54(4), 447-457.

Ratelle, C. F., Guay, F., Vallerand, R. J., Larose, S., \& Senécal, C. (2007). Autonomous, controlled, and amotivated types of academic motivation: A person-oriented analysis. Journal of Educational Psychology, 99(4), 734-746.

Reeve, J. (2002). Self-determination theory applied to educational settings. In E. L. Deci \& R. M. Ryan (Éds), Handbook of self-determination research (p. 183-203). Rochester, NY: University of Rochester Press.

Reeve, J., \& Jang, H. (2006). What teachers say and do to support students' autonomy during a learning activity. Journal of Educational Psychology, 98(1), 209-218.

Richard, G. (2015). Pratiques enseignantes et diversité sexuelle. Analyse des pratiques pédagogiques et d'intervention d'enseignants de l'école secondaire québécoise (thèse de doctorat, Université de Montréal, Canada). Consulté de http://hdl.handle.net/1866/11405

Richard, G., Chamberland, L., \& Petit, M.-P. (2013). L'inclusion de la diversité sexuelle à l'école: les enjeux pour les élèves lesbiennes, gais, bisexuels et en questionnement. Canadian journal of education, 36(1), 375-404.

Rivers, I. (2000). Social exclusion, absenteeism and sexual minority youth. Support for Learning, 15(1), 13-18. 
Roehrle, B., \& Strouse, J. (2008). Influence of social support on success of therapeutic interventions: A meta-analytic review. Psychotherapy: Theory, Research, Practice, Training, 45(4), 464-476.

Rosario, M., Schrimshaw, E. W., \& Hunter, J. (2005). Psychological distress following suicidality among gay, lesbian, and bisexual youths: Role of social relationships. Journal of Youth and Adolescence, 34(2), 149-161.

Rosario, M., Schrimshaw, E. W., \& \& Hunter, J. (2011). Different patterns of sexual identity development over time: Implications for the psychological adjustment of lesbian, gay, and bisexual youths. Journal of Sex Research, 48(1), 3-15.

Rosenberg, M. (1965). Society and the adolescent self-image. Princeton, NJ: Princeton University Press.

Rothman, E. F., Sullivan, M., Keyes, S., \& Boehmer, U. (2012). Parents' supportive reactions to sexual orientation disclosure associated with better health: Results from a populationbased survey of LGB adults in Massachusetts. Journal of Homosexuality, 59(2), 186200.

Rotter, J. B. (1990). Internal versus external control of reinforcement: A case history of a variable. American Psychologist, 45(4), 489-493.

Russell, C. J., \& Keel, P. K. (2002). Homosexuality as a specific risk factor for eating disorders in men. International Journal of Eating Disorders, 31(3), 300-306.

Russel, S. T., Seif, H., \& Truong, N. L. (2001). School outcomes of sexual minority youth in the United States: evidence from a national study. Journal of Adolescence, 24(1), 111127.

Ryan, C., Huebner, D., Diaz, R. M., \& Sanchez, J. J. (2009). Family rejection as a predictor of negative health outcomes in white and latino lesbian, gay, and bisexual young adults. Pediatrics, 123(1), 346-352.

Saewyc, E. M., Homma, Y., Skay, C. L., Bearinger, L. H., Resnick, M. D., \& Reis, E. (2009). Protective factors in the lives of bisexual adolescents in North America. American Journal of Public Health, 99(1), 110-117.

Savin-Williams, R. C. (1990). Gay ans lesbian youth : Expressions of identity. New York, NY: Hemisphere.

Savin-Williams, R. C. (1995). Lesbian and gay male and bisexual adolescents. Oxford: Oxford University Press.

Sheets Jr., R. L., \& Mohr, J. J. (2009). Perceived social support from friends and family and psychosocial functioning in bisexual young adult college students. Journal of Conseling Psychology, 56(1), 152-163.

Shilo, G., \& Savaya, R. (2011). Effects of family and friend support on LGB youths' mental health and sexual orientation milestones. Family Relations, 60(3), 318-330.

Schunk, D. H., \& Zimmerman, B. J. (2008). Motivation and self-regulated learning: theory, research, and applications. New York, NY : Routledge.

Spencer, S. M., \& Patrick, J. H. (2009). Social support and personal mastery as protective resources during emerging adulthood. Journal of Adult Development, 16(4), 191-198.

Tang, H.-B., Pu, W.-D., \& Yao, S.-G. (2008). A review of the relationship between social support and attachment. Chinese Journal of Clinical Psychology, 16(5), 551-553.

Teasdale, B., \& Bradley-Engen, M. S. (2010). Adolescent same-sex attraction and mental health: The role of stress and support. Journal of Homosexuality, 57(2), 287-309.

Vallerand, R. J., Blais, M. R., Brière, N. M., \& Pelletier, L. G. (1989). Construction et validation de l'Échelle de Motivation en Éducation (EME). Revue canadienne des sciences du comportement, 21(3), 323-349.

Warner, J., McKeown, E., Griffin, M., Johnson, K., Ramsay, A., Cort, C. et al. (2004). Rates and predictors of mental illness in gay men, lesbians and bisexual men and women: Results from a survey based in England and Wales. British Journal of Psychiatry, 185(6), 479-485.

Weibley, S. M. (2010). Creating a scale to measure internalized homophobia among selfidentified lesbians. Thèse de doctorat, The Johns Hopkins University, Maryland, USA.

Weinrich, J. D., \& Klein, F. (2002). Bi-gay, bi-straight, and bi-bi. Journal of Bisexuality, 2(4), 109-139. 
Zhao, Y., Montoro, R., Igartua, K., \& Thombs, B. D. (2010). Suicidal ideation and attempt among adolescents reporting "unsure" sexual identity or heterosexual identity plus same-sex attraction or behavior: Forgotten groups? Journal of the American Academy of Child \& Adolescent Psychiatry, 49(2), 104-113.

\section{RÉSUMÉ}

L'objectif du présent projet était d'examiner les associations entre le soutien de la famille et des amis, le rapport à l'orientation sexuelle, la détresse, le bien-être et le fonctionnement scolaire de jeunes lesbiennes, gais et bisexuel(le)s québécois $(n=29)$. Les résultats ont révélé que le soutien de la famille était négativement associé au rapport conflictuel à l'orientation sexuelle ainsi qu'à la détresse et positivement associé au bien-être. Le soutien des amis était associé négativement à la peur de la violence homophobe et positivement au bien-être. Le rapport conflictuel à l'orientation sexuelle était associé positivement à la détresse et négativement au bien-être. Le soutien des enseignants était positivement associé à la motivation scolaire et négativement à la violence homophobe, cette dernière étant négativement associée à la motivation.

\section{MOTS CLÉS}

LGB, soutien social, détresse, bien-être, orientation sexuelle, vécu scolaire, adolescents

\section{ABSTRACT}

The goal of the present project was to examine associations between social support from family and friends, feelings towards sexual orientation, distress, well-being, and school functioning in young lesbians, gays, and bisexuals from Quebec's Outaouais region $(n=29)$. Results revealed that family support was negatively associated with conflicted feelings towards sexual orientation, as well as with distress, and positively associated with well-being. Support from friends was negatively associated with fear of homophobic violence, and positively associated with well-being. Conflicted feelings towards sexual orientation were associated positively with distress, and negatively with well-being. Support from teachers was positively associated with school motivation, and negatively associated with homophobic violence at school, the latter being also negatively associated with motivation.

\section{KEY WORDS}

LGB, social support, distress, well-being, sexual orientation, school functioning, adolescents 